\title{
SOCIEDAD IBERICA
}

\section{DE BIOMECANICA}

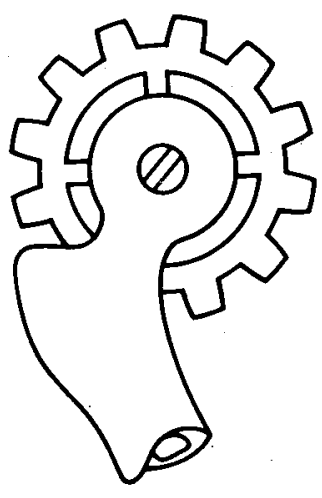

Reglamento de Congresos 
ARTICULO 1.․ LA S.I.B. celebrará:

a) Symposium.

b) Reuniones con Sociedades Nacionales o Internacionales afines.

El Symposium será de periodicidad anual y, preferentemente, en las mismas fechas. Se sugiere la primera semana de noviembre.

Las características de las Reuniones podrán ser revisadas cuantas veces sea necesario. La duración del Symposium anual será de dos días.

ARTICULO 2. El Symposium anual se celebrará en una localidad española que, a juicio de la Asamblea General, reúna las condiciones idóneas y en particular:

a) Existencia de una entidad que acoja el Symposium con dotación suficiente en número y capacidad de salas, así como áreas para las exposiciones científica y comercial, si así se requiriera.

b) Capacidad hotelera y facilidades de comunicación.

Las propuestas de sede se harán presentando a la S.I.B. una memoria documentada, con indicación del Presidente y Comité Organizador propuestos así como el presupuesto económico, como mínimo tres meses antes de la celebración de la Asamblea General, en la que se votará.

ARTICULO 3. La Junta Directiva de la S.I.B., creará una Comisión Técnica Asesora de Congresos. Esta comisión estará presidida por el Vicepresidente de la S.I.B. Esta, en colaboración con la Comisión Organizadora de cada uno de ellos se ocupará de su organización y velará por la adecuada gestión.

ARTICULO 4.․ En el Symposium Anual y Reuniones, las actividades científicas se organizarán en forma de Sim- posios, Mesas Redondas, Comunicaciones Solicitadas, Comunicaciones Libres, Talleres de Trabajo, Curso de Formación Continuada y Paneles de Discusión. Se presentarán Proyecciones Audiovisuales y se habilitará un espacio para la colocación de Carteles Científicos.

ARTICULO 5.․ Habitualmente habrá dos Temas Oficiales en cada Congreso, que se elegirán con un año de antelación y podrán presentarse en forma de Symposium o Mesas Redondas.

Los Miembros Numerarios tendrán derecho a solicitar la presentación de un Tema Oficial mediante una Memoria que deberá obrar en poder de la S.I.B. como mínimo un mes antes de la fecha de la Asamblea General en la que tendrá lugar su elección. En la Memoria constará el nombre del Director, colaboradores y centros participantes en su elaboración. La Memoria se dividirá en las siguientes partes: justificación, introducción, material y métodos, discusión y conclusiones, especificando el tiempo de duración y su forma de presentación (Simposio o Mesa Redonda). El tiempo de duración quedará finalmente supeditado a la organización del Congreso.

La Junta Directiva hará una selección de los temas propuestos y aquellos que sean seleccionados se pondrán a votación en la Asamblea General, en el curso de la cual el Director defenderá su Memoria. En caso de no haberse recibido ninguna Memoria dentro del plazo señalado o si las presentadas no tuvieran las características apropiadas la Junta Directiva queda capacitada para designar directamente el o los Temas Oficiales y su Director o Directores.

ARTICULO 6.․ Las Comunicaciones solicitadas podrán concederse presen- 
tando una petición razonada que deberá obrar en poder de la S.I.B. un mes antes de la Asamblea General en la que tendrá lugar su elección. Se aceptarán hasta un máximo de cuatro en cada Congreso.

ARTICULO $7 .^{\circ}$. Para que las Comunicaciones Libres sean admitidas habrá de enviarse un resumen completo a la Comisión Organizadora con un mes de antelación a la fecha de celebración del Congreso. La Comisión decidirá sobre la procedencia de su inclusión en el programa definitivo. Para la admisión definitiva de las Comunicaciones y su inclusión en el programa el presentador tendrá que estar inscrito previamente al Congreso. En el caso de que el autor de una Conferencia Libre no se presente para su lectura sin causa justificada queda excluido de la presentación de una Comunicación Libre en el Congreso siguiente.

ARTICULO 8.․ Será obligatorio presentar por escrito la ponencia o comunicación antes de la celebración del Congreso a la Comisión Organizadora.

ARTICULO 9.․ Los Simposios, Mesas Redondas, Comunicaciones Solicitadas y Comunicaciones Libres deberán tener un tiempo suficiente para su discusión.

ARTICULO 10.․ En todos los Congresos se podrá habilitar un espacio para la presentación y discusión de Carteles Científicos. Sus autores estarán obligados a explicar o aclarar su contenido a los congresistas interesados, según el horario que se indique en el programa del Congreso.

ARTICULO 11. ․ Los Paneles de Discusión y Talleres de Trabajo, serán programados por la Comisión Organizadora que facilitará el lugar y el horario para su celebración.

Se adoptará un procedimiento similar para las Exposiciones Científicas $u$ otras exhibiciones.

ARTICULO 12.․ La Comisión Organizadora en colaboración con la Junta Directiva de la S.I.B. podrá organizar Cursos de Formación continuada con matrícula independiente de la del Congreso para aquellos congresistas que deseen participar a los que se entregará una Certificación Acreditativa de su asistencia.

ARTICULO 13.․ Durante el Congreso se reservará el tiempo necesario para la celebración de la Asamblea General Ordinaria de la Sociedad.

ARTICULO 14. . Las inscripciones de los asistentes al Congreso se clasificarán en:

a) Congresistas Socios.

b) Congresistas pertenecientes a sociedades afines.

c) Congresistas no Socios.

d) Congresistas cooperadores.

ARTICULO 15.․ El superávit o déficit en su caso, que pueda producirse en la Tesorería de los Congresos organizados por la S.I.B. será absorbido por la propia S.I.B., previo estudio económico del Comité organizador aprobado por la Junta Directiva.

\section{DISPOSICION FINAL}

La Junta Directiva de la S.I.B., como máxima representante de la Sociedad, queda autorizada para interpretar aquellos puntos no expuestos en este Reglamento.

BAYONA, seis de noviembre de 1992 


\title{
Evolución filogenética de la bipedestación desde la biomecánica
}

\author{
AUTOR: DR. ALBERT ISIDRO \\ SERV. C. ORTOPÉDICA Y TRAUMATOLOGIA \\ HOSPITAL CENTRAL ALIANÇA/BCN
}

Dirección: Prof. Antoni Viladot

Universitat Autónoma de Barcelona

\section{RESUMEN}

La Bipedestación (BP) apareció hace más de 3,5 millones de años entre los periodos Plioceno Inferior y Mioceno, en algún representante del grupo de los Homínidos o tal vez algún Hominoide; pero con toda probabilidad relacionado con Austrolopithecus afarensis.

Se estudian en éste trabajo las diversas hipótesis que tratarian de explicar el porqué de la aparición de éste cambio locomotor. Hipótesis de tipo Bioclimático, Hipótesis de Rol, tanto sociales como reproductivas; Hipótesis Posturales e Hipótesis Bioenergéticas.

Se avanza una posible Cronofilogenia de la Bipedestación, a partir del Oligoceno, con la cadena evolutiva compuesta por Propliopithecidae, Proconsulidae, Kenyapithecus, Austrolopithecidae $y$ Homínidae.

Se citan los tipo de locomoción preadaptadores a la $B P$, extrapolando estudios realizados sobre primates actuales.

$Y$ finalmente se indican las principales adaptaciones anatómicas y su base biomecánica, empezando por la Cintura Pélvica, Rodilla, Tobillo y haciendo especial mención de todos y cada uno de los elementos que forman el pie. Para finalizar se hace referencia a los estudios realizados en las Pisadas de Laetoli, de una antigüedad aproximada de 3,7 M.a.

«Hemos nacido para perseguir la Verdad; poseerla corresponde a un Poder Superior»

MONTAIGNE

"La Bipedestación fue el primer cambio hacia la
Evolución Humana»
LAMARCK, 1809;
HAECKEL,1868;
DARWIN,1871

Somos una especie excesivamente egocéntrica. Nuestro antropocentrismo viene de lejos; en 1799, el médico inglés Charles White escribía: «Donde encon- traremos, como no sea en un europeo, esa cabeza noblemente arqueada que contiene tal cantidad de cerebro? Donde, esa variedad de rasgos y esa plenitud en la expresión, esas sonrojadas mejillas y esos labios de coral?

El Hombre, através de la Historia, se ha sentido orgulloso de 2 características, que nos diferencian del resto de animales:

1) Nuestra Alma

2) Nuestro «Porte»

Por desgracia y, como ya vaticinaba Sigmund Freud en el siglo pasado, nuestra relación con la Ciencia nos obliga a pagar un precio casi intolerable por cada parcela de Conocimiento. Así, la Astronomía, nos traslada desde el Centro del Universo, a un rincón marginal de nuestra galaxia. La Biología, nos humilla, aún más si cabe, cambiando nuestra posición desde un Simulacro de Dios a un Simio Erecto y Desnudo.

Una vez la Ciencia y la Humildad, nos han situado en el lugar que nos corresponde, podemos ver como aquellas características que tanto enorgullecieron a nuestros antepasados, fueron ya desarrollados con anterioridad por Natura.

Este sería el caso de nuestro «Porte». La Postura Erecta o Bipodalidad, así como el mecanismo de locomoción que de él se deriva o, que a él se llega, la Bipedestación; fueron utilizados con gran éxito desde hace más de 200 millones de años, durante la Era Mesozoica por los Dinosaurios.

Para Alan Charig, del Dpto. de Paleontología del British Museum y una de las mayores autoridades mundiales en Dinosaurios; para éstos Grandes Saururios uno de sus mayores logros, fue la capacidad de Bipedestar.

Los Dinosaurios se diferencian de otros Reptiles Arcaicos por poseer extremidades de tipo Columnar o sea, emplazadas directamente por debajo del cuerpo (circunstancia por la cuál disminuye el brazo de palanca durante la locomoción).

No obstante, la capacidad de Bipedestación (BP), apareció en un grupo de Reptiles Arcaicos, los Arcosauros, 
habitantes de ciénagas que dominaban el paisaje durante la transición entre el Pérmico Sup. y el Triásico Inf. (250 Ma. aprox.); y que, al contrario de otros reptiles eran Carnívoros (NORMAN, 1985), éste podría haber sido el caso de Lycaenops, un tetrápodo que a su vez podía desarrollar una locomoción BP.

La Paleoicnología o estudio de las Huellas Fósiles, no ha mostrado diversos conjuntos de huellas, en diferentes partes del mundo, que mediante su estudio (ALEXANDER, 1976; DEMATHIEU, 1986, etc), se ha podido demostrar la perfecta adaptación de diferentes familias de Dimosauros a la BP, así como también, se ha podido calcular la velocidad que pudieron desarrollar.

Por otro lado, el hallazgo y estudio de las piezas fósiles correspondientes a éstos Dinosaurios Bípedos, nos ha permitido saber que eran Digitígrados, cuyo peso recaía en 3 poderosos radios metatarsales medios, casi perpendiculares al suelo; mientras que el talus y calcaneo son muy poco significativos. Cabe destacar que las zonas de mayor carga durante la marcha son la art. interfalángica y la zona postero-sup de los MTT.

La Postura Erecta y la BP ocasional, son una práctica común en diferentes especies animales (ej. Oso); después de los anteriormente citados Dinosaurios y sus sucesores, tras la Extinción de éstos a finales del Mesozoico, las Aves.

Este tipo de postura y de locomoción están relacionadas con ciertas situaciones (agresión/defensa, vigilancia, rol), pero no comportan ningún tipo de adaptación morfológica.

La BP, tal como la entendemos actualmente, aparecerá como consecuencia de lasuma de unas determinadas circunstancias, en un periodo bastante puntual de la evolución de los Primates y probablemente a partir de una determinada especie.

La transición entre un Primate Antropoide no BP a un Homínido que si lo es, llevo consigo la incorporación de una serie de Adaptaciones Morfomecánicas en su Anatomía.

En éste trabajo se intentan analizar puntual y actualizadamente toda esta serie de circunstancias y cambios que desembocaron en la BP.

«Where, When, How Fast and From What, Did Man Emerge?»

\section{ESQUEMA 1 \\ FILOGENIA DE LOS PRIMAATES}

PURGATORIUS

PLESIADAPSIFORMES

HAPLORRINOS

PROTOSIMIOS

\section{CATARRINOS}

$\checkmark$ AEGYPTOPHITECUS

PROCONSULIDAE

DRYOPITHECIDAE

KENYAPITHECUS

PRE-AUSTROLOPITHECIDOS

AUS.afarensis

$\triangle$ aUS.africanus

HOMO habilis

AUS. robustus

HOMO erectus

AUS. boisei

HOMO sap.arcaic

HOMO sap.neandertal

HOMO sap.sapiens
$70 \mathrm{Ma}$

$60 \mathrm{Ma}$

$30 \mathrm{Ma}$

$15 \mathrm{Ma}$

$5 \mathrm{Ma}$

$3.5 \mathrm{Ma}$

$3 \mathrm{Ma}$

$2 \mathrm{Ma}$

$1 \mathrm{Ma}$

\section{FILOGENIA DE LOS PRIMATES}

Este esquema representa un avisión global de la Evolución de los primates, desde su primer representante, Purgatorius ceratops (Cretácico Sup. Nontana/U.S.A., hasta Homo sapiens.

Las cirras de la derecha corresponden a la secuencia cronológica aproximada de aparición de las diferentes familias y géneros, oscilando entre los 70 millones de años (MA) para Purgatorius, hasta $1 \mathrm{Ma}$. que corresponde a la interfase entre Australopithecus.

Boisiei y Homo sapiens arcaic.

La columna superior izquierda, es la cadena evolutiva de Prosimios y Simios; en la columna medio central se encuentran Simios y Póngidos de tendencia antropoidea, desde Aegyptopithecus del Mioceno Medio de Al-Fayum/Egipto hasta Kenyapitecus, con toda probabilidad el primer Hominoide.

Los Hominidos debutan con Aus.Afarensis, y divergen en 2 grandes familias. Australopithecus y Homo.

En nuestro país los restos más antiguos corresponden al grupo Adapidae (entre plesiadapsiformes y Haplorrinos).

PD.- Las letras en cursiva corresponden a Géneros, mientras que el resto representan Familias. 
Para una mejor comprensión del fenómeno global que comportaría el desarrollo de las $\mathrm{BP}$, diremos, que la importancia otorgada a los animales que la desarrollaron, fue ya prevista por Lynneus, 1758 , el cual en la $X^{\text {a }}$ Ed. de su obra Systema Naturae, introduce el vocablo Primate (que proviene del latín «Primas», que significa: el que está en primer lugar). Asimismo en el Esquema $n^{\circ} 1$ viene descrita una aproximación a la Filogenia de los Primates.

\section{HIPOTESIS DESENCADENANTES DE LA BP. (PICKFORD, 1989)}

\section{A. HIPOTESIS BIOCLIMATICAS}

Esta serie de hipótesis o teorías tratan de explicar la aparición de la $\mathrm{BP}$, en base a un Cambio Climático que conlleva un cambio del Ecosistema; durante la Fase Potencial o PreaAdaptativa que desembocaría en la BP.

Se fundamentan muchas de éstas hipótesis en una Singularidad Climática especial denominada Crisis Mesiniense que tuvo lugar durante el Mioceno Sup. hace aprox. 6,5 Ma; durante la cual se conjugaron un descenso térmico importante y un periodo de sequias estacionales.

Dichos cambios pudieron presionar sobre un determinado grupo de Antropoides, que eran formas «potencialmente bípedas».

Dentro de éste grupo de hipótesis bioclimáticas cabe destacar la Hipótesis de Divergencia Geográfica o EastSide Story (COPPENS, 1983), la cual desarrolla sus elementos en base a un cambio en el ecosistema determinado por la Fosa del Rif, la cual discurre por el Africa del Este, desde Moçambique hasta Siria en su extremo norte. Esta formación geológica determinaría, por el cambio climático anteriormente mencionado, una zona húmeda al Oeste, compuesta de selva tropical y otra zona mucho más árida al Este, afectada por las sequias estacionales y cuyo paisaje predominante serían la savana.

Este cambio, de selva tropical a savana comporta un Incremento en el Horizonte Visual con la posibilidad de ejercer una mayor acción predadora a la vez que se esta más sometida a ella. (OAKLEY, 1954; HOWLS, 1959; LEAKEY, 1961; DAY, 1986).

La cada vez mayor escasez de árboles, obligaría a estos primates preadaptados, a efectuar trayectos más largos en suelo firme Open-Ground Transforest Travel (SUSMAN, 1985).

Se la suma también la dispersión de los recursos alimenticios, ya no se está en una selva con gran cantidad de frutas y hojas; esto no se está en una selva con gran cantidad de frutas y hojas; esto conlleva la Necesidad de Transporte(HEWES, 1961), debido al progresivo aumento en las distancias para conseguir la misma cantidad de alimentos (ZILHMAN \& BRUNKER, 1979; RODMAN \& McHENRY, 1980; ROSE, 1984).

La adecuación y especialización de la Vegetación y de la Fauna a éste nuevo ambiente, conduciría probable- mente a un cambio radical en la Dieta, volviéndose cada vez más Omnívora: por una parte Granívoro, dada la gran cantidad de gramíneas salvajes que comporta la savana arbustiva; condición ésta favorecedora de la pinza del pulgar y la consiguiente liberación de los brazos (VALVERDE, 1967). El cambio que representó la condición de Carroñero, ya que el mono preadaptado y/o el Hominoide no podía ser un cazador eficaz, por lo cual estaba obligado a seguir los grandes rebaños de ungulados que deambulaban por la savana y espearía a que algún cazador eficaz diera caza a alguno de éllos, para luego competir por los despojos con los demás carroneros. Hunter and Scavanging Hipt. (TUTTLE, 1981; CARRIE, 1986; SINCLAIR, 1986).

Otra de las teorías basada en los cambios climáticos, es la que propone el Incremento del Grado de Insolación, mucho mayor es una savana en una selva; establece la disminución de la superficie corporal expuesta al sol, a medida que el sol va subiendo en el horizonte. Para un individuo $\mathrm{BP}$ el área expuesta al sol es máxima durante el alba y mínima cuando el sol ésta en su zenit, lo cual en un hominoide que, además se está quedando sin el pelaje tupido que había poseido, la radiación solar es menor si este individuo está en posición bipodal que si continúa siendo tetrápodo. (WHEELER, 1984).

\section{B. HIPOTESIS DE ROL}

Estas hipótesis teorizan sobre los condicionantesde la Vida en Grupo o Social presentes en un grupo de monos antropoides avanzados puede desarrollar «per se» la BP.

Dentro de éstas hipótesis de rol diferenciaremos 2 grupos:

\section{Estrategias Sociales.}

Es el caso, anecdótico por cierto, de la teoría de la Desaparasitacióin Corporal (MORRIS, 1967).

\section{Estrategias Reproductivas o Sexuales.}

Que citan como condicionantes más destacables, la divergencia reproductiva entre Póngidos y Hominoides. Los Póngidos o Grandes Monos Antropomorfos tienen un patrón reproductivo Epigámico,el cual no solo no varía sino que se ve reforzado en lo Hominoides. También la Estrategia Reproductora hacia la «K» (o sea, un hijo cada mucho tiempo, y sometidoa unos cuidados maternales prolongados) en los Póngidos; a una involución hacia la estrategia «r» (menor periodode tiempo entre 2 alumbramientos) en los Homínidos.

Estos factores reproductivos en conjunción con el ecosistema predominante nos llevaría a 2 situaciones esenciales para el desarrollo de la BP; el Transporte Unimano de Hijo y el Transporte de Comida.(LOVEJOY, 1981; GALLUP, 1982).

\section{HIPOTESIS POSTURALES}

Este tipo de teoría desembocan en la consecución de una Postura Pronograda, cada vez más frecuente. 
Como hemos visto anteriormente, la postura erguida se desencadena en diversas situaciones, como podían ser: la posibilidad de efectuar una Caza al Acecho en un antropoide omnívoro «Wait and Pounce Hipt» (EICHMAN, 1987) o la «Geist Stalking Model» (MERKER, 1984). La Sedestación Erguida, también se arguye como factor importante en el desarrollo de la postura pronograda, habitualmente adoptada durante la comida en grupo «Joly's Feeding Hypt» (PILBEAM \& JACOBS, 1979; LEUTTENEGGER, 1987).

Las actitudes de Intimidación o Amenaza tanto en situaciones defensivas como ofensivas, situación parecida a la anteriormente mencionada en los Osos (DART, 1959; DAY, 1986).

Finalmente mencionaremos, a modo anecdótico, la rocambolesca teoría de Vadeo o «Acquatic Model of Life» (HARDY, 1960), por la cual la postura pronograda derivaría del vadeo de zonas lacustres en los trayectos de los antropoides.

\section{HIPOTESIS BIOENERGÉTICAS}

La BP. es una forma de locomoción Eficaz para las necesidades de desplazamiento en los Homínoides o antropoides preadaptados, teniendo en el cambio en su Ecosistema.

La BP, objetivamente, ha demostrado ser más eficaz y conferir más resistencia en su forma humana, que la BP ocasional practicada por la mayoría de Póngidos. (RODMAN\& McHENRY, 1980). Los autores citados valoraron en un estudio comparativo entre el Gasto Energético de las locomociones en diversos tipos de Primates.

Se calculó el Consumo Previsto en $\mathrm{ml} \mathrm{02/gr/Km}$. (en función del peso total y de las distancias medias diarias observadas por diferentes autores) y lo compararon con el Consumo Real observado a una velocidad media de $2,9 \mathrm{Km} / \mathrm{h}$.

De los resultados así obtenidos, el más llamativo corresponde a la diferencia entre el Consumo Previsto y el Consumo Real en los casos de BP, tomando como referencia a Pan (Chimpanze) y al Homo Sap; pues Pan representaría un $149 \%$ de consumo previsto $(0,351$ a $0,522)$ y en Homo que resultó ser del $86 \%(0,225$ a $0,193)$.

Resultados que nos demuestran que la BP en el hombre es bioenergéticamente más favorable (consume menos), circunstancia que se observa principalmente durante la Marcha, no siendo así en la Carrera. Fenómeno que refuerza las hipótesis que atribuyen a la BP. una gran utilidad en los grandes desplazamientos. (HEWES, 1961; ZILHMAN, 1979; RODMAN 1980).

\section{CRONOFILOGENIA DE LA BIPEDESTACION}

Desconocemos con exactitud Cuando apareció la BP como forma predominante de locomoción en algún tipo de Hominoide Preadaptado.
Este desconocimiento actual es debido a que en la Evolución de los Primates hay un «Agujero Negro» en el devenir evolutivo que condujo a los Hominoides en Homínidos. Esta zona obscura se localza en el Mioceno Sup. y abarca un periodo comprendido entre los 14 y los $4 \mathrm{Ma}$. aprox.

Para darnos una idea de la escasez de restos fósiles catalogables como Hominoides durante este periodo, mencionaremos que hasta 1988 , se tenía constancia solo de 14 piezas de las cuales, únicamente 3 , correspondían a estructuras relacionadas de forma directa con la BP. (HILL 6 WARD, 1988), estas son:

Fémur Proximal / Middle Awash: Maka (Ethiop) < $4.0 \mathrm{Ma}$. Fíbula Distal / Sahabi: P 33A (Libia)

Talus /Muruyur (Tanzania) $5.0 \mathrm{Ma}$ $14.0 \mathrm{Ma}$.

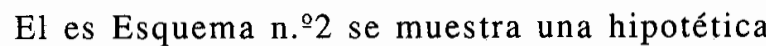
Evolución Cronológica hacia la BP. a partir de Formas Potenciales.

Iniciamos nuestra serie cronológica en el periodo Oligoceno con la apariciónde Aegyptopithecus zeuxi, estudiado por SIMONS\& MEYERy perteneciente al yacimiento de Al-Fayum en Egipto. Este pequeño simio, tenía como características diferenciales, en lo que respecta a su modo de locomoción y, con respecto a otros primates coetáneos; la capacidad de Suspensión Arborícola (SUSP) y la capacidad de Trepar (CL.); por el contrario no se encuentran rasgos que indiquen la capacidad para el Salto (LEAP).

Durante el Mioceno Inferior aparece un tipo de simio, Proconsul (major, africanus o nyanzae), descubierto en la Isla Rusinga en el interior de lago Victoria / Kenia, por LOUIS \& MARY LEAKEY? 1948; el cual conserva su capacidad CL., pero su quadrumanismo arborícola (Q.ARB) se convierte en una locomoción con las palmas y plantas de sus extremidades, tanto en el suelo firme como en el árbol.

En el Mioceno Medio aparece Kenyapithecus wickerii, al que podíamos catalogar como primer Hominoide en la evolución de los primates, éste, puede desplazarse en suelo firme sin perder su capacidad CL.

Durante la «zona obscura» que corresponde al Mioceno Sup., nuestro particular y desconocido ancestro, pudo desarrollar una BP ocasional y muy limitada, mientras que con el paso del tiempo fue incrementando muy lentamente su frecuencia, perfeccionando paulatinamente ésta para obtar a una forma de locomoción eficaz.

Con la irrupción de los Homínidos en el Pliopleistoceno, cuyo representante más antiguo corresponde a Austrolopithecus afarensis, > 3,5 Ma., la BP se constituye por primera vez la forma predominante en el repertorio locomotor, sin perder capacidad CL.

Para finalizar la serie cronobiológica con Homo habilis, coetáneo, durante más de un millón de años, con diversos tipos de autrolopithecus, y que únicamente es BP. (SENUT, 1989) 


\section{ESQUEMA 2 \\ CRONO-FILOGENIA DE LA BIPEDESTACION}

OLIGOCENO MIOCENO inf MIOCENO med MIOCENO SUP PLIOCENO

$38 \quad 24$

18

125

1.2

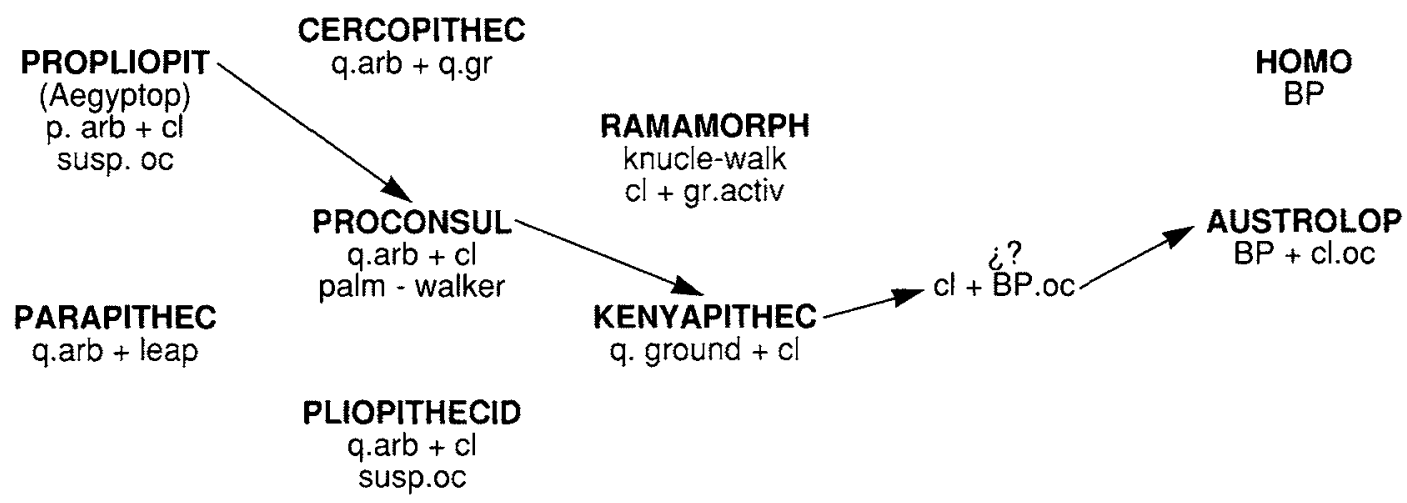

\section{CRONONOFILOGENIA DE LA BIPEDESTACION}

Este esquema trata de correlacionar, en el tiempo, diversos géneros y familias de primates con su correspondiente repertorio locomotor, encadenados para la consecución de la locomoción bípeda (BP).

Distribuidos en los diferentes períodos de aparición, quedan dispuestas las familias o géneros más representativos de primates. Debajo del nombre, y abreviado, aparecen las características de locomoción.

En negrita y encadenado mediante flechas, se observa la posible cronología y filogenia.

La cadena empieza en el oligoceno con Propiopithecidae (Aegyptopithecus) - Proconsulidae Henyapithecus - zona obscura del Mioceno Sup. - Austrolopithecidae - Hominidae en el Plio-Pleistoceno.

\section{ABREVIATURAS}

q. Arb:

q. ground:

susp. occ:

leap:

$\mathrm{cl}$ :

palm-walker: cuadrumano arboricola.

cuadrumano en suelo firme. suspensión ocasional. saltador arborícola. trepador.

palmigrado arborícola/terrestre.
Gr. activities: Knucle-walker: bp:

actividades manuales en suelo firme. pongid-like locomotion. bipedestación.

Basado en textos de Brigitte SENUT, 1989.

\section{FORMAS PREADAPT ADORAS DE LOMOCION HACIA LA BP.}

En la gran diversidad que conforma el mundo de los Primates y en particular de los Simios, diremos que coexisten, en un mismo individuo, diferentes patrones de locomoción, presentando, en relación del nicho ecológico que habita; una forma predominante que utiliza con más frecuencia.

Esta forma predominante de locomoción viene determinada por el ecosistema en que habita, como ya hemos mencionado $\mathrm{y}$, muy directamente por el tamaño del simio.

Interrelacionando los tipos de locomoción observados en los primates y simios actuales $y$, teniendo en cuenta su posible cronobiología, mencionaremos las diversas hipótesis con las que se trató de predecir la apariencia de nuestros «potenciales» ancestros, comparándoles con los primates actuales.

En 1940 JONES identificaría a los Tarsídios como nuestros hipotéticos ancestros (los tarsidios no son Simios); en 1948 HOORTON, defendió la hipótesis de que nuestros ancestros no podían ser otros que los Póngidos (nuestros actuales primos, genéticamente hablando); en 1968 WASHBURN, agregó a lo anteriormente teorizado por Hoorton, la condición de desarrollar la Knucle-walking, como sistema predominante de locomoción (actualmente se considera a la knuckle-walking como una forma de locomoción que permite al póngido desplazarse por el suelo sin que por ello, su mano pierda adaptaciones arborícolas). 
En nuestros días se tiende a considerar que el aspecto que debería tener nuestro predecesor, podría corresponder a un Hylobatido (gibón, etc.) de entre 9 y 13,5 Kg. de peso. (TUTTLE, 1981).

Las características locomotoras que éste hipotético precursor debería runir, se centrarían en:

Como signos positivos: Braquiación (BRAC) y $\mathrm{Cl}$. vertical (VCL)

Como signos negativos: No quadrúmano arborícola (Q. ARB), así como no desarrollar en su extremidad sup., ningún tipo de adaptación a la deambulación en suelo firme.

\section{«La Braquiación preadaptó a nuestros ancestros a la Postura Erecta»}

Keith, 1923; Morton, 1926.

La braquiación comporta, como principal variante con los demás tipos de locomoción dentro del mundo de los primates, una Extensión Completa y Pasiva de la Cadera; postura muy anormal en cualquier Tetrápodo. Esta extensión mantenida, poseería unos efectos sobre estructuras osteoarticulares. (FEAGLE,1981)

Por lo que respecta a la capacidad de Trepar Verticalmente o Vertical Climbing (V.CL), diremos que durante su ejecución, 1 a disposición del Tórax queda por encima de la Pelvis; apareciendo asociada una Lordosis lumbar. Su ejecución activa comporta una rotación medial del Anillo Pelviano; una rotación interna del Muslo; y una extensión, esta vez Activa, de la Cadera y de la Rodilla.

La variación anatómica más precoz en simios que efectúan este tipo de locomoción es la disminución de la distancia entre el Sacro y el Acetábulo, lo cual desmultiplica las cargas y reduce la lateralización de la columna en cada paso. (característica observada ya en algunos primates europeos del Mioceno como Orotiphecus bambolii. Toscana/Italia).

Estas características en la locomoción han sido estudiadas en el individuo vivo y en su medio natural. También han sido extrapoladas y reforzadas por medio de estudios de laboratorio, centrados principalmente en técnicas como el E.M.G. y los Globogramas, de los cuales se puede objetivar la similitud entre los movimientos desarrollados durante la denominada «Slow V.Cl» y la $\mathrm{BP}$ humana. Por el contrario se han analizado las profundas diferencias que existen entre nuestra BP y aquella practicada ocasionalmente por los póngidos (ej. pan, gorilla, etc.) (PROST, 1980)

La Velocidad de Transición para que formas preadaptadas no BP iniciarán con cierta frecuencia la locomoción $B P$ varía según autores.

Para algunos (TUTTLE, 1969; LOVEJOY, 1981), ésta transición fue relativamente rápida, puesto que ya estaban preadaptados.

Para otros (ROSE, 1984; JAANUSSON, 1986), la transición fue en forma de «Mosaico», en el cual mien- tras algunos individuos eran ya BP., otros se encontrarían todavía en la fase potencial.

\section{«Adam est collectif»}

\section{YVES COPPENS}

\section{ADAPTACIONES MORFOMECANICAS DEL TREN INFERIOR}

La BP. no apareció como una particularidad de cierto grupo de Simios determinada por el Azar.

La BP. puede considerarse como la evolución «más lógica» en un determinado grupo de simios antropoides y/o Hominoides, para desplazarse en un terreno y un clima cambiante.

La deambulación bipeda trajo consigo el desarrollo de toda una serie de adaptaciones en la globalidad del cuerpo. No obstante la zona anatómica que debió sufrir cambios más acusados fue el Tren Inferior, entendiendo éste como la zona anatómica comprendida entre la zona lumbar y el pie.

El primer Homínido conocido (A. Afarensis), practicaba ya predominantemente la $\mathrm{Bp}$., pero todavía no había tenido tiempo para adaptarse a ella; por lo cual'su eficacia no era equiparable a la BP del Hombre Moderno; aunque en el caso de Aus. Afarensis, su necesaria adaptación, estaba ligada al Instinto de Supervivencia. Creo esclarecedora la frase de JUNGERS, 1976, que habla de «el Dramático alargamiento de las piernas surge como uno de los mayores cambios de Aus. Afarensis en su evolución hacia el Hombre Moderno».

WOLPOFF, 1983, en una carta de réplica publicada en la prestigiosa revista Nature; aporta unos excelentes trabajos biométricos para evaluar la relación, extrapolada, de las longitudes de fémur con respecto a una serie de parámetros lumbares, obtenidos de diversas muestras de Austrolopithecus y otras entre los que cabe citar a Homo Sap. Neandertalensis y a Homo Sap. Sapiens (pigmeos y amerindios).

Los resultados son elocuentes: mientras que la longitud, en términos absolutos, del fémur de Austrolopithecus (Al.288.1/Lucy y STS.14) es mucho menor que en las demás muestras; la relación entre la longitud del fémur/altura L3 y la relación entre la mencionada long.fémur/altura total de las vértebras lumbares, se obtienen unas cifras muy parecidas y en algún caso está dentro del rango humano, no sólo en el caso de los Pigmeos, si no que también en individuos de talla normal, como sería el caso de los Amerindios.

Como Características Anatómicas Generales en lo que respecta al conjunto formado por Anillo PélvicoCadera-Fémur, que de un modo significativo se encuentran relacionadas con el Grado de BP. mencionaremos:

- Disminución Altura Iliaco

$++t$

- Desplazamiento Post-Sup. Espina Iliaca +++ 
- Pilar Iliaco Unico

- Canal Músculo Psoas-iliaco

- Canal Musc. Obturador Externo

- Genu Valgo

- Canal Femoropatelare Profundo

- Labio Troclear Cóndilo Externo (modif STERN 6 SUSMAN, 1983

1983)

A la derecha de cada característica general y, mediante un sistema de valoración por cruces, se ha incluido su presencia o no, en Aus. Afarensis.

Las características puntuales que presenta la PELVIS y que están relacionadas directamente con el grado de BP., las podríamos dividir en:

a) Angulo Sacro: formado por la intersección de líneas trazadas a partir de las caras anteriores de S1 y S5. Este parámetro está rełacionado con el grado de BP. alcanzado. (Ver tabla $n^{\circ} 1$ )

De ella se desprende que AL (Aus. Afarensis) con un ángulo sacro de 31.5 , posee una cifra extrapolable a la capacidad de BP. en un niño de 4 años.

b) Modificaciones en Os Innominatum o Coxal: Citaremos la ya mencionada expansión del Ala Iliaca, una pronunciada Escotadura Ciática, una Espina antiinf. prominente. Junto con el fenómeno de la expansión del ala ilíaca, deberemos mencionar que la ala ilíaca en los primates no antropoides es de situación Posterior, en los Homínidos se desplaza hasta una localización Posterolateral, para hacers Lateral en Homo.

c) Angulos de Torsión Pelviana: En ellos se relacionan los distintos planos tridimensionales que adoptan ilión/isquion/sacro/acetábulo, correlacionándoles con el tipo evolutivo del primate a que pertenezcan. Tabla $n^{\circ} 2$

d) Pilares Pelvianos y Musculatura Extrínseca: La función del principal músculo extrínseco, el Glúteo mayor, varía entre los Primates y Homínidos, pasando deser Abductor + Rotador Ext. a Extensor + Rot. Ext. en Homo (TUTTLE, 1975). El Hombre pose un único Pilar Pelviano que separa al grupo muscular compuesto por, el Glúteo medio y menor del tendón de la Fascia Lata. Por el contrario AL. posee 2 pilares pelvianos. (LOVEJOY,1973; ARSUAGA,1980).

El estudio singular de la CADERA lo iniciaremos centrándonos en las variaciones del Acetábulo* desde el punto de vista articular.

La Fascies Lunata o zona articular del acetábulo para el caput femoralis, presenta en Homo un Cuerno Anterior muy marcado, lo cual repercute en el índice entre la altura de la superficie articular con el diámetro máximo que, mientras en Homo es de 0,98, en AL.288.1 es de 0,78 , debido a la carencia significativa del cuerno anterior, cuyo significado biomecánico se centra en la posibilidad de una hiperextensión de la cadera, mientras que su falta, como es el caso de AL, nos indica la presencia de un flexo de cadera.

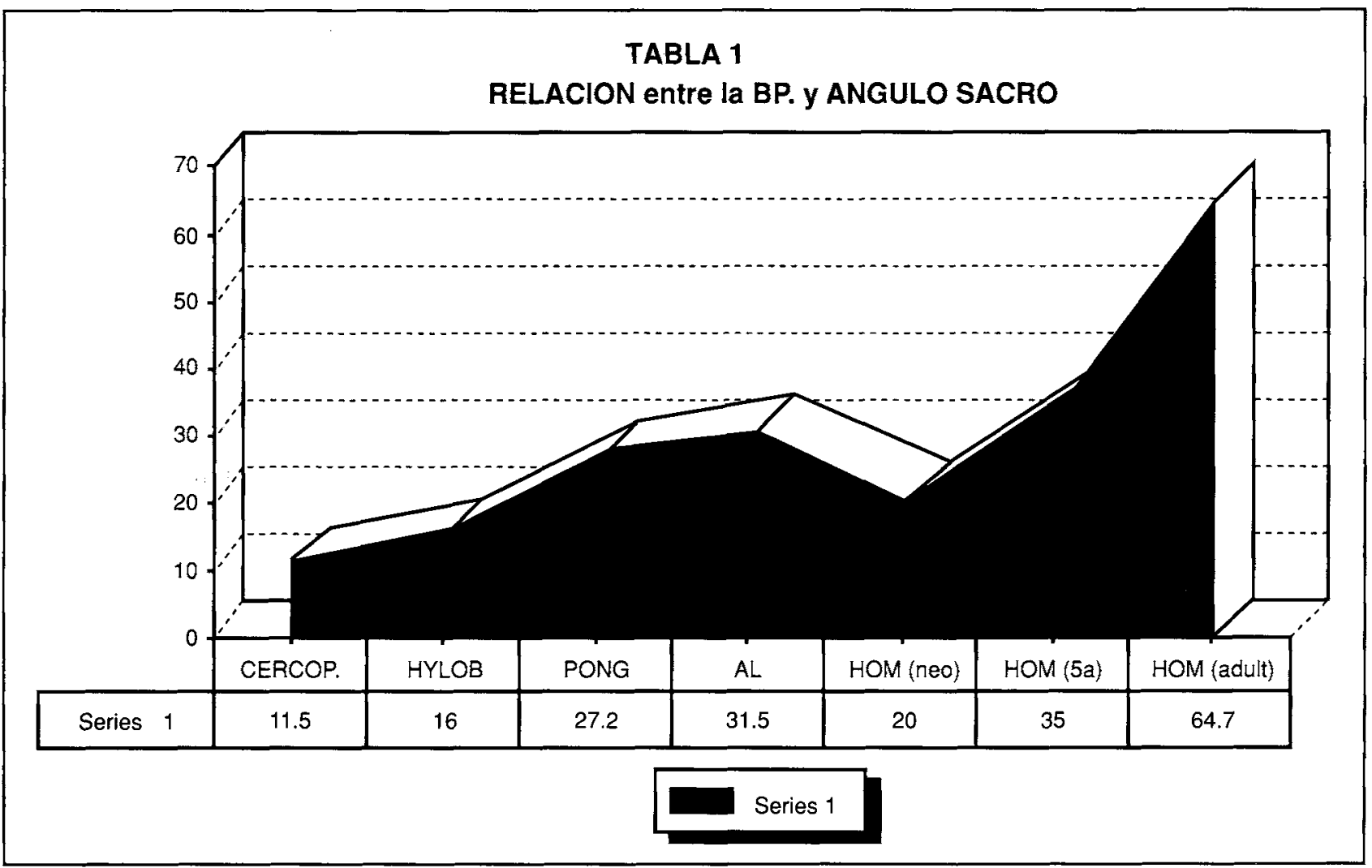


TABLA N. 22

MODIFICACIONES PELVICAS

ANGULOS DE TORSION (AT)

\begin{tabular}{cccccc}
\hline \multicolumn{1}{c}{$\boldsymbol{A}$ AT. } & $\begin{array}{c}\text { PRIMAT } \\
\text { NO-ANTROP. }\end{array}$ & STS14. & SK3155. & HOMO. & AUTOR \\
\hline ILIO/SACRO & - & $42.3^{\circ}$ & $45.0^{\circ}$ & $31.2^{\circ}$ & $\begin{array}{c}\text { (BROOM, 58 } \\
\text { MCHENRY, 75) }\end{array}$ \\
ILIO/ISQUION & $100^{\circ}$ & $86 / 5^{\circ}$ & - & $65 / 6^{\circ}$ & (CHOPRA, 62) \\
ILIO/ACETAB. & $100^{\circ}$ & $82.5^{\circ}$ & $75 .^{\circ}$ & $79.8^{\circ}$ & (MCHENRY, 75) \\
\hline
\end{tabular}

Del Caput Femoralis* y la región más craneal del fémur* diremos que, como diferencias más significativas entre Homo y AL: el aumento de volumen de la cabeza, una disminución en la longitud del cuello femoral y un mayor volumen en el trocánter mayor.

Importante, por su significado biomecánico, es el estudio de los límites que corresponden a la superficie articular del caput femorals; éste límite, en Homo, se prolonga hacia el trocánter mayor a través de su margen antero-sup (característica de hiperextensión de cadera, complementaria a la presencia del cuerno anterior de la fascies lunata). Por el contrario en Pan (chimpanze), esta línea discurre por el margen postero-sup., significando un mayor grado de flexión en la cadera.

Por lo que respecta a AL., el límite articular es casi paralelo a la línea del trocánter mayor.

La presencia en AL.333.3 de un canal para el músculo Obturador Externo, atestigua el grado de desarrollo de la Bp., al ser éste rotador externo de la pierna.

El Angulo Cérvico-diafisario, de cuya conformación resulta el grado de valgo o varo de la cadera, sitúa a AL. dentro de rango humano de valguismo $\left(121^{\circ}-133^{\circ}\right)$; en AL. $288.1\left(123^{\circ}\right)$ y en AL.333.3 $\left(125^{\circ}\right)$.

La Diáfisis Femoral presenta el Angulo Femorobicondíleo, causante de la oblicuidad diafisaria del fémur y por consiguiente del componente femoral del genu valgo.

La rodilla humana presenta un valguismo que se cifra entre los $9^{\circ}$ y los $11^{\circ}$; en austrlopithecus el valguismo es aún más acusado (AL. 129.1 $=14.5^{\circ} / \mathrm{STS} .34=15^{\circ} /$ TM. $1513=14^{\circ}$ ).

Fenómeno paradójico si medimos el ángulo en el resto de póngidos los cuales tienden a $0^{\circ}$, o sea al varo de rodilla.

E1 Surco Femoro-Patelar es profundo, tanto en Homo como en $\mathrm{AL}$, su función es la de potenciar la capacidad extensora del cuadríceps, capacidad que viene complementada por la Forma Elipsoide del Condilo Externo Femoral, la forma elipsoide en este condilo externo solo está presente en Homo, mientras que algunos ejemplares de AL. (Al.333.4 / TM.1513), quedarían dentro del rango de variación humana en su zona inferior. (McHENRY, 1986).

Por otra parte, la Hipertrofia del Labio Externo del Condilo Femoral, refuerza la profundidad del surco femoro patelar, impidiendo la luxación externa de la rotula en una rodilla cada vez más valguizada (BRATTSTROM, 1964).

La tendencia progresiva hacia la consecución de un Genu Valgo, permite al pie a situarse directamente por debajo del Centro de Gravedad durante la Fase Monopodal de la marcha bípeda.

«Don (D. Johanson), de cuclillas, bajo un sol abrasador, intentaba encajar aquellos dos huesos y de pronto comprendió, aquello no era un mono, era un Ser Humano».

MAURICE TAIEB

\section{ARTICULACION TALO-CRURAL (COMPONENTE TIBIAL Y FIBULAR)}

Zona de especial importancia para la evolución de la BP., zona compuesta por las superficies articulares distales de Tibia y Fíbula.

Caracteres como la Inclinación de la Superficie Articular de la Tibia Distal; la forma de la Troclea Talocrural y la situación de diversos Ejes de Movilidad que de esta articulación se desprenden, son componentes importantes del engranaje adaptativo que, en esta zona puntual, dio paso a la $\mathrm{BP}$.

Iniciaremos nuestro estudio por la conformación de la SUPERFICIE ARTICULAR INFERIOR de la TIBIA de la que destacaremos especialmente su Angulo de Inclinación en el plano sagital; parámetro importante para la deducción del arco móvil y de la direccionalidad del eje talo-crural.

Este ángulo se forma por la intersección del eje paralelo al suelo que pasa por la zona más distal de uno de los 2 pilares tibiales, anterior o post., con la línea que los une.

El ángulo será positivo en el caso de que el Pilón Ant. sea el más distal o prominente y negativo si lo fuera el Pilón Post. (ver Tabla n 3.) 
De esta tabla se desprende un fenómeno muy remarcable, a mi modo de ver, que es el singular momento adaptativo en el que se encuentra sumida esta zona articular, si comparamos los distintos individuos de austrolopithecus, dado que, mientras AL.288.1 nos da una cifra de $-5^{\circ}$, lo cual nos hace suponer un alto grado de flexión dorsal del pie, característica ligada a la vida arborícola; en AL.333.6 el ángulo de inclinación es de $+5^{\circ}$ muy cerca de la cifra hallada en Homo $\left(+7,9^{\circ}\right)$, el cual presenta una flexión dorsal del pie muy limitada. (STERN/SUSMAN, 1983).

Las variaciones del Angulo formado por la Tibia en los límites Máximos de Flexión Dorsal y Ventral, sitúa el rango humano entre los $35^{\circ}$ y $\operatorname{los} 60^{\circ}$; a Gorilla entre $37^{\circ}$ y $58^{\circ}$; a Pan entre $40^{\circ}$ y $68^{\circ}$; mientras que para AL.288.1 su ángulo es de $64^{\circ}$ (LATIMER, OHMAN, LOVEJOY, 1987).

Se puede predecir, de estas cifras que el arco máximo que recorre la diáfesis tibial, es inversamente proporcional al tamaño del animal. Así el máximo arco móvil corresponde a «Lucky» con $64^{\circ}$ ( $1.10 \mathrm{~m}$ y $30 \mathrm{Kg}$.), siendo mínimo en Gorilla. (1.80 m y $165 \mathrm{~kg}$ ). (BIEWENER,1983).

La característica más destacable por lo que respecta a la Fíbula Distal, se centra en la diferencia de Inclinación del Límite Sup. de la Sindésmosis T/P Distal.

En Homo éste límite sup. discurre de antero-sup. a postero-inf.; mientras que en AL. va de antero-inf. a postero-sup.

Configuración que se relaciona con el resto de estructuras que forman la mortaja tibio-peronea astragalina, importante biomecánicamente, en Homo, durante la dorsiflexión del pie.

\section{PROCESOS EVOLUTIVOS DEL PIE / GENERALIDADES}

El pie, en su conjunto, es la zona anatómica que más ha debido evolucionar, tras la consecución de la locomoción BP.

Antes de describir, los cambios ocurridos en los principales componentes del Retropie y en las Falanges; daremos una visión en conjunto de las diferencias más significativas, de determinadas zonas puntuales, realizadas mediante Anatomía Comparada entre, los Simios en general con los Monos Antropoides y, éstos últimos con respecto a los Homínidos.

Por lo que respecta a las diferencias básicas entre Simios y Monos Antropoides, cabría destacar:

\section{ZONA ANATOMICA SIMIO M. ANTROPOIDE \\ Articul. Medial Talus Grande Pequeña}

Articul. Lateral Talus Oblicua Con Escalón Lat.

Posición Canal Musc.

Flexor Hallucis Longus Lateral Ext. Medial

Tubérculo Peroneal 1 MTT Grande Pequeño

Longitud Relativa

Conjunto Huesos Tarsianos Largos Cortos

De especial importancia en estudios paleoantropológicos de piezas procedentes de excavaciones y que suelen ser muy parciales, destacaremos la posición del Canal de paso para el Músculo Flexor Largo del Hallux, que nos determina, con bastante precisión, el momento evolutivo en el que nos encontramos; pues varía de una

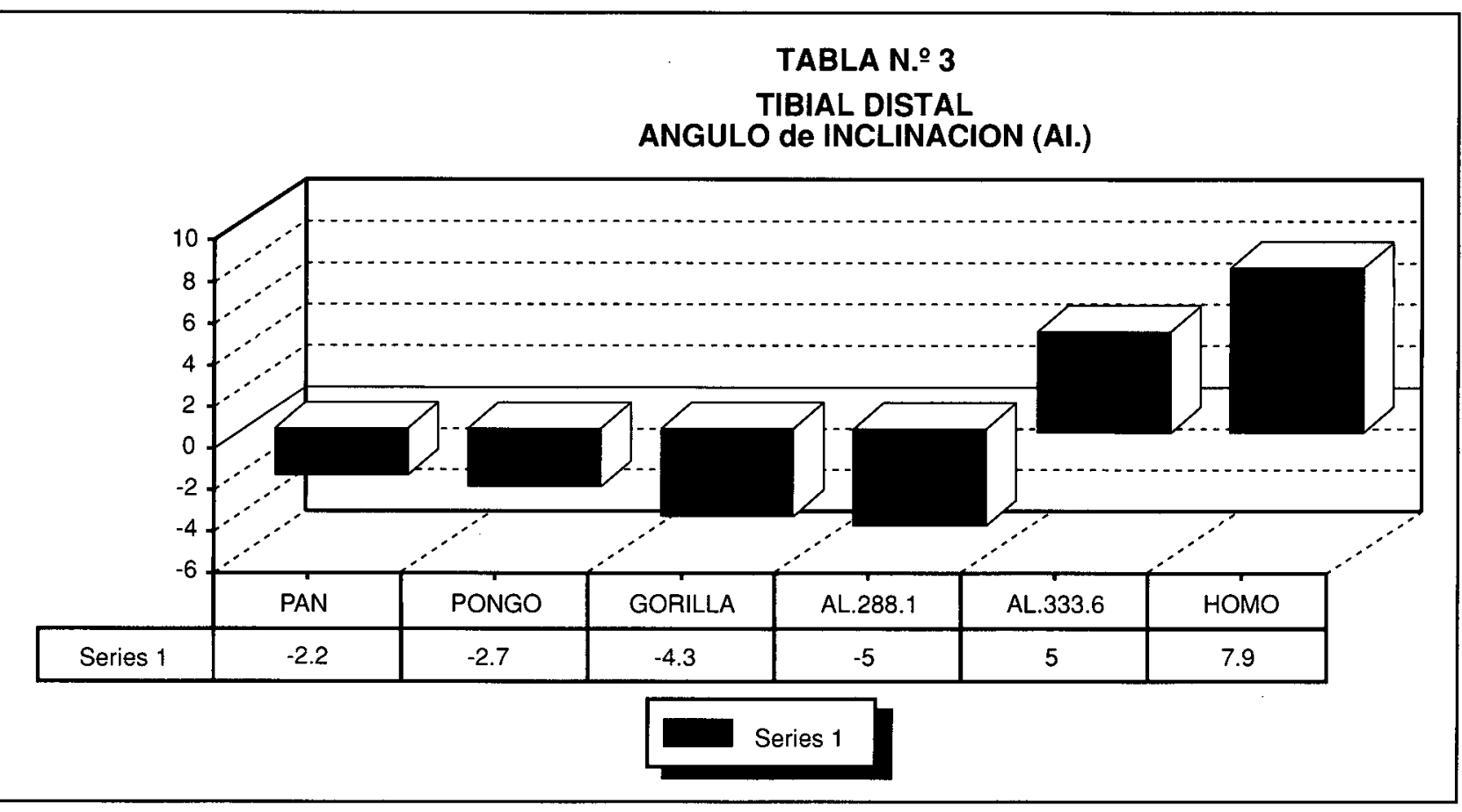


posición lateral externa en los Simios, pasa a ser más medial en Monos Antropoides y se coloca en una posición interna en los Homínidos. (actualmente se está utilizando este parámetro para el estudio de un fragmento distal de tibia atribuido a Dryopithecus laietani, encontrado en el yacimiento de Can Llobateras en el Vallés/BCN MOYA, GIBERT, ISIDRO-LLORENS del Institut de Paleontología M. Crusafont/Sabadell).

Mucho más compleja resulta la diferenciación de zonas puntuales del conjunto del pie entre, Antropoides Primitivos y Homínidos.

Para ello tomaremos como referencia una parte del exhaustivo trabajo de GEBO, 1989.

\section{ANTROPOIDES PRIMITIVOS/ HOMINIDOS}

- Angulación medial + rot. cuello y cabeza del Talus

- < angulación acortamiento cuello T.

- Calcáneo largo y estrecho principalmente su zona ant.

- Calc. acortado en zona ant. + alarg. zona media.

- Ausencia de Tubérculo Plantar

- Tubérculo Plantar

- Eje calcáneo-cuboideo suave.

- Eje cal. cuboideo profundo

- Navicular o Escafoides largo.

- Acortamiento Navicular

- Cuboides largo

- Acortamiento Cuboides

- Articul. plana en el 1 MTT

- Articul entocuneifore

-Hallux corto.

- Alargamiento del Hallux

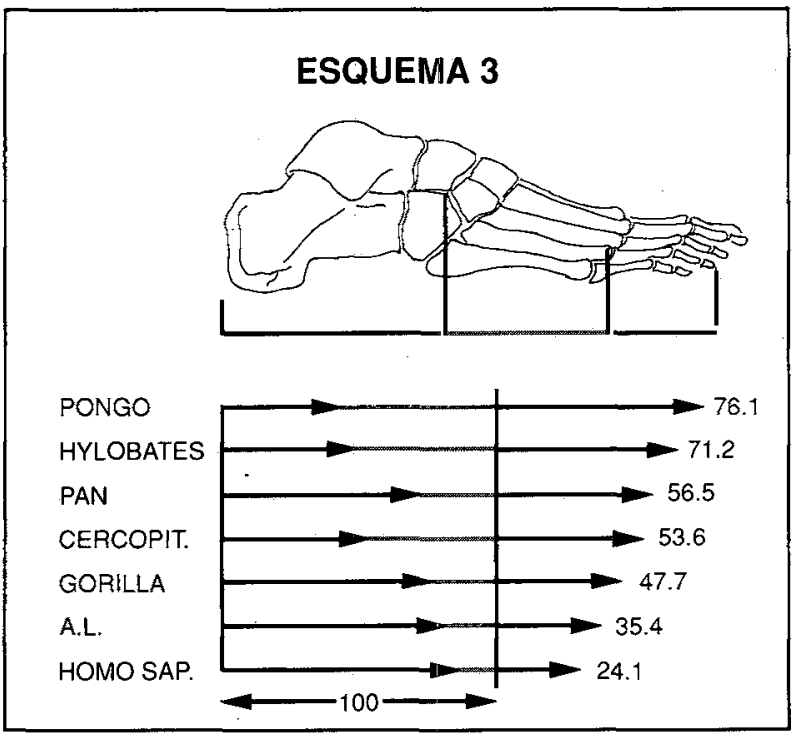

Son significativas tambien las longitudes relativas comparadas de las zonas del retropie, zona medial del pie y falanges que aparecen en ESQUEMA $\mathrm{N}^{\circ} 3$, en la cual se expresa una modificación del gráfico aparecido en los trabajos de WHITE \& SUWA, 1987, y en el cual se puede observar la comparación de la longitud relativa de las falanges de diversos Simios, es comparada a la longitud 100 , correspondiente al conjunto de longitudes del retropie y de la zona medial del mismo.

Podemos ver como la longitud relativa de las falanges de AL., que representa un 35\% del total del resto del pie, es la medida más similar a la que posee Homo $(24,1 \%)$; parametros ambos muy alejados a los observados en Pongo (orang-utang) que alcanza un $76,1 \%$.

\section{ESTUDIO SINGULAR DEL RETROPIE}

\section{A) TALUS O ASTRAGALO}

El Talus ha sufrido gran número de modificaciones, tanto en lo que respecta a su morfología, como en las superficies articulares que presenta en su conjunto.

Aegyptopithecus, Oropithecus y otros simios ancentrales que componen nuestra linea filogenética, poseian un talus cuya distancia máxima* se encuentra situada en el plano transversal o lo que es lo mismo muy largo, contrapuesto a la relativa poca altura. Concomitante con esta característica podemos observar que el grado de divergéncia hacia medial*, entre los ejes troclear y el trazado por la linea media del cuello del talus, es muy elevado, en contraposición a aquel que se observa en Homo que es casi paralelo.

El arco correspondiente a la Maxima Superficie Articular* de la troclea varia segun los tipos de simios y oscila entre los $125^{\circ}$ (R 110/145 ) en Pan; a los $110^{\circ}$ (R 90/125 ) en Gorilla; y $140^{\circ}$ en AL. 288.1. Parametros estos que deben ser comparados a los de Homo que cifrados en $112^{\circ}(\mathrm{R} 95 / 120)$.

También es interesante destacar en estudios paleoantropológicos el denominado Angulo Apical*, parámetro relacionado también con la zona troclear y que es debido a la diferéncia de longitud entre los labios o crestas trocleares, interna y ext, caractéristica en cada Simio.

En el hombre moderno el labio interno o medial es paralelo al eje mayor del pie y ligeramente más largo que el labio externo el cual diverge, condición ésta que unida con la morfología de la articulación sindesmal comporta una reducción de la flexión dorsal del pie.

Este ángulo de forma por la intersección de las lineas trazadas uniendo las zonas más anteriores y posteriores de ambos labios trocleares. Este ángulo apical en Homo es algo negativo al ser más largo en labio interno que el externo por lo que el ángulo se forma en la zona externa del talus; no es así en los demás antropoides, en los cuales el labio interno es mucho más corto que el externo, asi Gorilla posee un a. apical de $+40^{\circ}$ aprox., mientras que AL. 288.1 se situa en $+14^{\circ}$. (LATIMER, LOVEJOY, 1987). 
El Límite Anterior de la Superficie Articular* correspondiente a la troclea talar, en Homo, queda truncada debido al freno o brida que produce la mortaje tibioperoneo-astragalina durante la dorsiflexión del pie. En contraposición los póngidos y en AL., el margen o límite anterior articular sobrepasa distalmente la base del cuello talar.

\section{B) CALCANEO}

\section{Foot») \\ «The Calcaneous is the Morphologycal Kernel of the} WEIDENREICH, 1923

El Calcaneo es sin lugar a dudas el elemento del pie, cuya transformación mas a contribuido al desarrollo de la BP.

El Calcaneo en el hombre moderno soporta, en posición bipodal, cerca del $80 \%$ del peso corporal, y ello se debe principalmente a la existencia de unos arcos en la boveda plantar y al aumento de volumen de su región posterior. (VILADOT).

Tomando como referéncia los trabajos que sobre el calcaneo en AL. efectuaron LATIMER \& LOVEJOY 1989; el análisis evolutivo que ha sufrido el calcaneo en 4 puntos que, a mi modo de ver, son definitorios para la adaptación a la BP.

\section{Incremento de la Tuberosidad Posterior}

Con respecto al incremento en el volumen en esta zona podemos observar que está en relación directa con el grado de BP y con la postura erecta TABLA N ${ }^{\circ} 4$.

De ella se puede extraer que, a pesar de su enorme corpulencia Gorilla adulto, alrededor de $165 \mathrm{Kg}$, posee tan sólo un Vol. de $27,3 \mathrm{cc}$ el cual contrasta con el de AL. 333,55, que con un peso en torno a los $35 \mathrm{Kg}$ el vol. de la tuberosidad post. se cifra en $29,8 \mathrm{cc}$.

Con respecto a la superficie coronal (cross section), correspondiente a la sección mínima de la tuberosidad post. los resultados obtenidos varian entre los $2,8 \mathrm{~cm} 2$ (R 2/3,8) en Pan; 4,7 cm2 (R 2,5/6,5) en Gorilla; $7 \mathrm{~cm} 2$ en AL. 333,8 y AL. 333,55, hasta llegar a Homo que posee una sección mínima media de $8,8 \mathrm{~cm} 2$ (R $6,5 / 11,8)$

El factor morfológico adaptativo que más influyó en el incremento de la Tuberosidad Posterior fue la aparición del

\section{Proceso Latero Plantar (PLP)}

Esta es una prominécia ósea latero-externa que se forma entre el Proceso Latero Medial, que poseen todos los póngidos en el calcaneo posterior, y la formación conocida como Troclea Peroneal.

La formación del PLP., incrementa en aproximadamente un $150 \%$ la superficie de contacto del calcaneo durante la fase talograda de la marcha bípeda.

El análisis biomecánico es muy claro: durante la marcha el pie se mantiene pronado debido a la suma del genu valgo y de la horizontalidad del eje talo-crural. Esta pronación conlleva una rotación externa del retropie sobre su eje sagital.

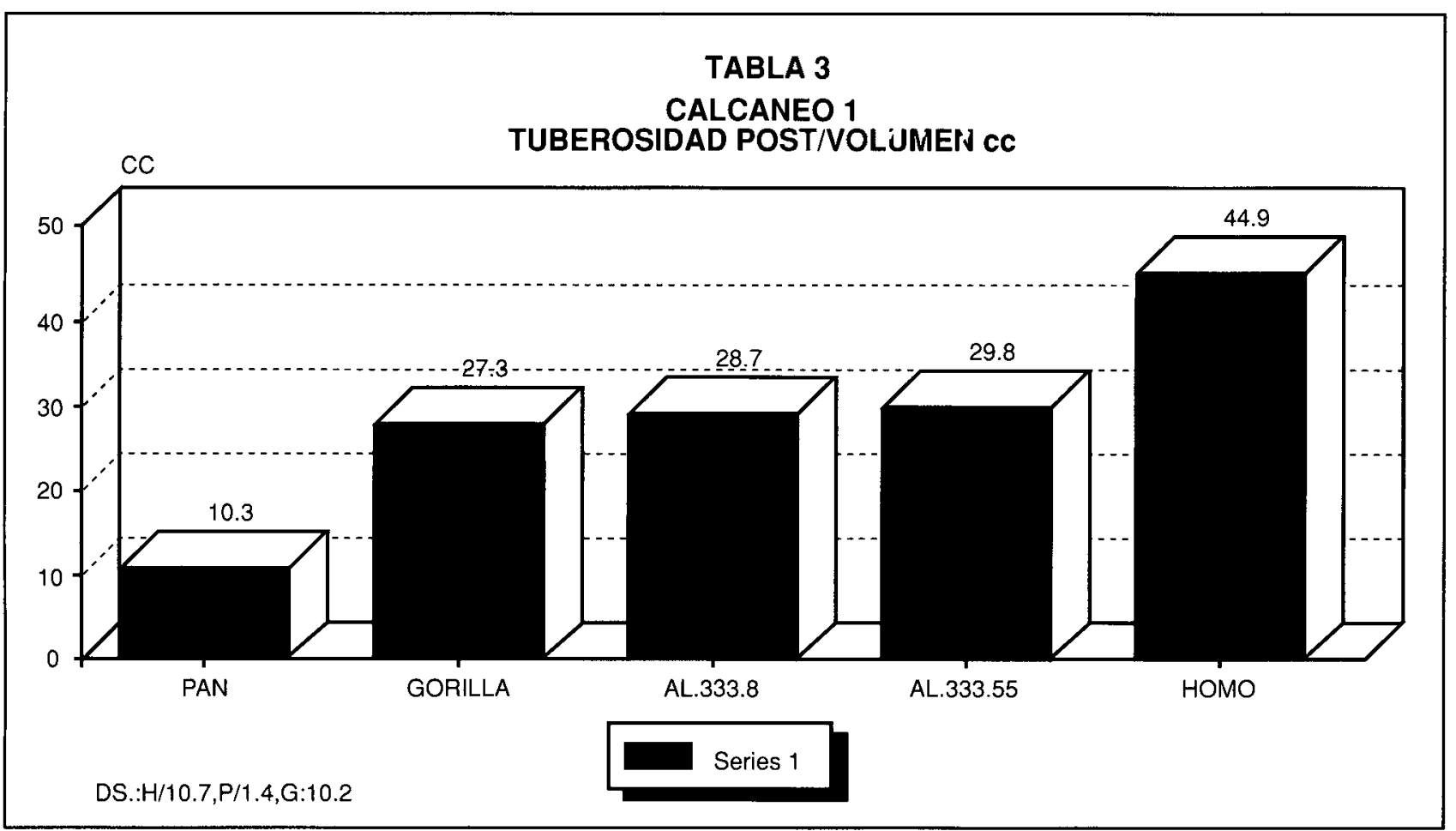


La preséncia del PLP. descarga del máximo stress al proceso latero medial (área demasiado reducida) durante la fase de apoyo talar de la marcha.

El PLP. se encuentra sólo en AL y Homo.

\section{Troclea Peroneal. (TP)}

La TP, es un canal óseo por donde discurren los tendones de los Músculos Peroneos.

Como consecuéncia del pronunciado talo varo del retropie en los póngidos, es necesario un canal para mantener en su posición y evitar así la luxación de los tendones peroneos.

Cuando la tuberosidad posterior del calcáneo se adapta a la marcha bípeda y aparece el Proceso Latero Plantar, éstos tendones peroneos quedan encajonados entre un maleolo peroneal más voluminoso y la pared externa del calcaneo.

Por lo que se deduce que a mayor PLP menor TP. Mientras que la coexisténcia de ambas formaciones óseas en una misma pieza puede significar que pertenezca a un Hominoide. (SUSMAN, 1984).

\section{Complejo Articular Subastragalino}

En Homo complejo articular subastragalino se compone de 3 superficies articulares. La diferéncia en el número es significativa, según la especie de primate estudiada:

Con 2 carillas art:

Aegyptopithecus zeuxi: Oropithecus bam. Proconsul africanus; Hylobates; Pan; Pongo.

Con 3 carillas art.:

Pliopithecus; Papio; Homo etc.

Desde un punto de vista biomecánico lo más importante no es el número total de superficies articulares, sino su disposición tridimensional en el espacio.

La horizontalidad de las superficies art. subastragalinas indicaría un incremento en las solicitaciones mécanicas a éste nivel, cuyas causas desencadenantes son 2 principalmente: un incremento de peso o la práctica habitual de la BP.

Esta horizontalidad lleva asociada una disminución del movimiento articular.

En Homo se conjuntan, en ésta zona, dos características debidas a la presencia de la BP: > radio zona articular y $<$ Angulo de Sustentación

$\begin{array}{lll}\text { Género } & \text { Radio art. en mm } & \text { Angulo Sustentación } \\ \text { Pan } & 14,2 \text { (ds 3.1) } & \left.110^{\circ} \text { (ds 9.3 }\right) \\ \text { Gorilla } & 20,8 \text { (ds 3.3) } & \left.100^{\circ} \text { (ds } 8.2^{\mathrm{a}}\right) \\ \text { AL. } & 24,5 & 82^{\circ} \\ \text { Homo } & 26,3 \text { (ds 3,2) } & 78,5^{\circ}\end{array}$

«Las Articulaciones Subastragalinas presentan diferencias entre los Póngidos y el Hombre y son de gran importancia para analizar el desarrollo de la Evolución Humana»

MORTON, 1924

\section{ANALISIS DE LA BOVEDA PLANTAR}

La estructura de la Bóveda Plantar y de los Arcos que la conforman son de gran importancia en la compresión de los fenómenos de repartición de cargas entre las distintas zonas del pie.

LAMY, 1986 efectua un riguroso estudio de paleoanatomia comparada centrado en el analisis de los elementos que conforman la boveda plantar.

En él son definidos 3 Arcos Plantares, que a continuación se mencionan junto a las estructuras activas y pasivas que la conforman y su filogénia:

$\begin{array}{lll}\begin{array}{ll}\text { Arcos } \\ \text { A. Longitudinal }\end{array} & \begin{array}{l}\text { Estructuras } \\ \text { T. Musc. Peroneus } \\ \text { Longus }\end{array} & \text { Filogénia } \\ \text { A. Transversal } & \text { id } & \begin{array}{l}\text { Hominidos } \\ \text { Fascia Plantaris }\end{array} \\ \text { A. Medio Longitud } & \text { Fóngidos y } \\ & \text { Fascia Plantaris } & \text { Hominidos } \\ & \text { Lig. Calcaneo-Navicular } & \text { Homidos }\end{array}$

Consideramos de especial importancia al Ligamento Calcaneo Navicular Plantar, especialmente su zona enthesal a nivel de la zona antero-inferior medial del Talus.

Los signos que indican la presencia de éste ligamento en el talus, demuestra la posible conformación del Arco Medio Longitudinal, formación ésta intimamente relacionada con la Postura Erecta.

En ningun Póngido aparecen signos de la zona de inserción de este ligamento; signos que empiezan a aparecer muy discretamente en AL. 288,1 y KNM.ER 1464 (correspondiente a austrolopithecus afarensis, y robustus), para estar perfectamente definido en el talus de OH.8 (correspondiente a Homo habilis).

\section{ANALISIS DEL ANTEPIEUUU}

El antepie en su conjunto está formado por la MTT y las falanges.

Han sido realizados diversos estudios biométricos de estas estructuras, como los realizados por SZALAY, 1986 a propósito de un exahustivo en medidas absolutas y relativas, Indices de Robustez, Indices de Aplanamiento Diafisario en los MTT; comparativamente entre el ya citado Oropithecus vs. papio, pan, pongo, etc.

Hemos de hacer mención, en éste apartado, de la histórica recopilación descriptiva de los huesos de pie, extraidos de la formación de Hadar (Ethiopia), durante las campañas que van desde 1974 y 1977, todos ellos pertenecientes a Aust. Afarensis (LATIMER, LOVEJOY, JOHANSSON, COPPENS, 1982)

De estos trabajos se desprende el progresivo acortamiento del conjunto anatómico que forman MTT y cuñas, así como el acortamiento de las falanges Esquema $\mathrm{N}^{\circ} 3$, tanto por lo que respecta a Homo, como en AL. 
Por lo que respecta al Primer Radio, esta va aumentando de longitud, haciendose cada vez más anterior o sca aduciéndose.

Las falange correspondientes a los radios más externos, tambien disminuyen de longitud y, de gran importancia, se van incurvando.

STERN \& SUSMAN, 1983 efectúan mediciones del radio de las falanges más próximales ( $1^{\mathrm{a}}$ falange) correspondientes a diferentes Simios y sus resultados son:

\section{$2^{\circ}$ dedo:}

Bonobo $/ 185^{\circ}$; Pan $/ 145^{\circ}$; Gorilla $/ 140^{\circ}$; Homo $/ 105^{\circ}$, AL. $333,93 / 150^{\circ}$; AL. $333,19 / 175^{\circ}$; AL. $333,57 / 180^{\circ}$

$3^{\circ}$ dedo:

Bonobo $/ 150^{\circ}$; Pan $/ 135^{\circ}$; Gorilla/ $125^{\circ}$; Homo $/ 103^{\circ}$; AL. 333,24/110 ; AL. 333,63/190

$4^{\circ}$ dedo:

Bonobo $/ 175^{\circ}$; Pan $/ 140^{\circ}$; Gorilla $/ 135^{\circ}$; Homo $/ 125^{\circ}$; AL. $333,93 / 150^{\circ}$; AL. $333,19 \S 175^{\circ}$; AL. 333,57/180

$5^{\circ}$ dedo:

Bonobo $/ 220^{\circ}$; Pan $/ 190^{\circ}$; Gorilla $/ 160^{\circ}$; Homo $/ 135^{\circ}$; AL. $333,62 / 245^{\circ}$

Para éste estudio se han tomado como parámetros objetivos, el radio del circulo cuya circunferéncia para por el punto medio de la art. MTT, un punto medio difisário y el punto más distal de la cabeza de la falange. según la fórmula: $1 / \mathrm{R} \times 10.000$.

En éste nivel cabria hacer una recopilación de las principales lineas de directrices seguidas en éste trabajo. Hemos mencionado una série de hipótesis por las cuales pudo haberse desarrollado la BP, una posible cronología, sus formas preadaptativas dentro del mundo de los simios, para finalizar haciendo un estudio más o menos exhaustivo de Anatomia Comparada.

No obstante, nos podríamos preguntar si existe alguna prueba palpable de que algunos Homínidos eran realmente bipedos. Y ésas pruebas, poseerían la suficiente antiguedad para reforzar el conjunto de teorias que dieron paso a la BP.

La respuesta es positiva y sus pruebas son:

\section{LAS PISADAS DE LAETOLI}

El yacimiento de Laetoli se encuentra en las inmediaciones del Rio Garusi, a unos $30 \mathrm{Km}$ del conocido yacimiento de Olduvai en Tanzania. Ambas zonas estudiadas por los equipos de LEAKEY y LUDWIG KOHLLARSEN en la década de los 30.

No fue hasta 1976, cuando un visitante de las excavaciones dirigidas en aquella época por MARY LEAKEY; ANDREW HII.L, quien tuvo la oportunidad de sacar a la luz, el denominado Tufo 7 o capa 7 , en la cual se podian contemplar numerosas huellas que, en un principio fueron atribuidas a algun tipo de Hominoide (LYONS, 1978), aunque posteriormente (TUTTLE, 1984) adjudicara su paternidad a algún tipo de úrsido.
Sin embargo, el más espectacular hallazgo en la zona del Tufo 7, se localizó en la Localidad 8, el 24 de Julio de 1978 gracias a PAUL ABELL, concretamente en el denominado Sitio G (a escasa distáncia de donde se había encontrado un resto parcial de maxilar humo en 19874). Se trataba de una huella correspondiente a la impresión de un Pie Izquierdo, de características totalmente compatibles con las humanas.

Posteriores estudios han descubierto y analizado un total de 39 huellas de homínido (G1 y G2, huellas humanas en paralelo, correspondiente a un individuo adulto y a un niño; mientras que las G3 corresponden a huellas sueltas), con una longitud total de $23,5+6$ metros; conservadas, maravillosamente, en una capa de ceniza volcánica (Volcán Sadiman, extinto) con un espesor que oscila entre los 12 y los $15 \mathrm{~cm}$.

Como es de suponer, han sido numerosos los estudios dedicados a éstas pisadas, entre ellos los que han propuesto un diagnóstico diferencial con otras posibles entidades causantes de tales improntas. Durante algún tiempo se mantuvo que las Pisadas de Laetoli podrían corresponder tanto a un Póngido como a un Homínido. A continuación haremos una comparación entre las características compartidas con póngidos, de aquellas exclusivamente humanas:

\section{Como características comunes destacaremos:}

- Una profunda Impresión Calcanea.

- Transferéncia de la carga através del borde externo.

Como características únicamente humanas:

- Aducción y posición anterior del Hallux.

- Longitu y curvatura estimada de las falanges.

Si observamos con detenimiento las pisadas del grupo G1, podremos advertir 3 zonas de presión que corresponden a:

- Pulpejo de Hallux.

- Cabeza del Primer MTT.

- Tubérculo Posterior del Calcáneo.

En un minucioso trabajo (WHITE \& SUMA, 1987), trataron de incluir, tras extrapolar el tamaño, posibles conformaciones de distintos pies correspondientes a Pan troglodytes, Austrolopithecus afarensis, Homo habilis y Hombre Moderno.

Se tomó como referéncia obligada, tras adecuar los distintos tamaños a la superficie de la huella, los 3 puntos de máxima presión arriba citados.

Tras efectuar las distintas reconstrucciones, se contató, que el pie que más se acercaba, en un alto grado, al de la muestra correspondía al de «Lucy» (A. Afarensis).

Circunstáncia, que nos permite cerrar el Círculo, por el cual se tenía, de una parte, huellas de homínido correspondientes al Plioceno cuya estratigrafia oscilaba entre los 3,49 y los 3,76 Ma. (LEAKEY \& HARRIS, 1987); por otra, la certeza de que los únicos homínidos que habitaban nuestro planeta en ése periodo eran exclusivamente, A. Afarensis. 
Por lo cual, tras análisis fotométricos se llega a la conclusión de que las Pisadas de Laetoli, fueron provocadas por Homínidos que, transmitían su peso durante la marcha, de manera muy similar a la del Hombre Moderno. (DAY, 1985).

Reconsiderando todos los aspectos discutidos anteriormente, podemos decir, no sin cierto orgullo, que Andamos Erguidos hace más de 3,5 Ma; pero a pesar de esé enorme periodo de tiempo, parece no haber sido suficiente para acabar de adaptar nuestra morfologia a éste tipo de postura y locomoción.

Patologías tan frecuentes como la artrosis de columna en general y en especial de sus zonas más móviles (cervical y lumbar); las ptosis viscerales; las insuficiéncias valvulares venosas (varices) y la compleja patología del pie, asi lo demuestran.

Con toda probabilidad y como lo demuestran observaciones en tribus primitivas cuyo pie, siempre ha permanecido descalzo; en auséncia de calzado, el pie tiende hacia una mayor flexibilidad y a un cavus.

Lo que el futuro depara para nuestro pie es a la vez lógico e imprevisible, por más que tendamos hacia un Pie Egipcio, con una predomináncia acusada del Primer Radio y una involución progresiva de las falanges en los radios más externos (GONZALEZ-CASANOVA \& VILADOT, ), nuestra Raza Humana es capaz, tendiendo hacia lo imprevisible, de convertir lo díficil en imposible.

«Creo haber encontrado el eslabón intermedio entre el animal y el hombre..., somos Nosotros»

K. LORENZ

\section{BIBLIOGRAFIA}

1. Abitol, M. Evolution of the Sacrum in Hominoids. Am. J. Phys. Anthropol. 1987; 74: 65-81.

2. Abitol, M. Sacral Curvature and Supine Posture. Am. J. Phys. Anthropol. 1989; 80: 379-389.

3. Alexander, R. McN. Estimation of Speeds of Dinosaurs. Nature. 1976; 261: 129-130.

4. Arsuaga, J.L. Iliac Angular Measurements in Austrolopithecus. J. Human Ev. 1981; 10: 293-302.

5. Asfaw, B. Proximal Femur Articulation in Pliocene Hominids. Am. J. Phys. Anthropol. 1985; 68: 535/538.

6. Christie, P.W., Ridley, J.N. Matematical Analysis of Articular Surfaces: Aplicacion to the Anatomy of Early Hominoids Ankle Joint. Hominidae: Proceedings of the 2nd Inter.Congress of Human Paleontology. 1989;; 61-66 Ed. Jaca Books/milan.

7. Coppens, Y. Le Singe, L'Afrique et l'Homme. Ed. Fayard/Paris, 1983.

8. Darwin, Ch. The Descent of Man. London 1871

9. Day, M. bipedalism: Pressures, Origins and Modes. In

Major topics in primate and Human Evolut. Cambridge Univ. Press. 1986; 188-202.

10. Demathieu, G.R. Nouvelles Recherches sur la Vitesse des Vertebres, auteurs de Traces Fossiles. Geobios. 1986; 19: 3 327-333.

11. Fleagle, J.G.; Stern, J.T.; Jungers, W.L.; Susman, R.L.; Vangor, A.K.; Wells, J.P. Climbing: A Biomechanical Link with Brachiation and with Biopedalism. Symp. Zool. Soc. London. 1981; 48: 359-375.

12. Gallup, G.G.; Suarez, S.D. Optimar Reproductive Strategies for Bipedalism. J. Human Ev. 1983; 12: 193/196.
13. Gebo, D.L.; Simon, E.L. Morphology and Locomotor Adaptations in the Foot in Early Oligocene Anthopoids. Am.J. Phys. Anthopol. 1987; 74: 83-101.

14. Gebo, D.L. Locomotor and Phylognetic Considerations in Anthropoid Evolution. J. Human Evol. 1989; 18: 201-233.

15. Hewes, G.W. Foot Transport and the Origin of Hominid Bipedalism. amer. Anthropol. 1961; 63:687/710.

16. Hill, A.; Ward, S. Origin of the Hominidae: The Record of African Large Hominoid Ebolution between $14 \mathrm{My}$ and 4 My. Yearbook of Physical Anthrop. 1988; 31: 49-83.

17. Hoorton, E.A. Up from the Ape. McMillan Ed./New York 1946.

18. Howells, W. Mankind in the Making: The Story of Human Evolution. Doubleday \& Co. Garden City. Ed/NY 1959.

19. Jaanusson, V. Balance of the Head in Hominid Evolut. Lethaia. 1986; 20: 165-176.

20. Jenkins, F.A. Chimpanzee Bipedalism: Cineradiographic Analysis and Implications for the Evolut.of Gait. Science. 1972; 178: 877-879.

21. Johanson, D.; Edey, M. Lucy. The Beginnings of Humankind. Simon \& Schuster Ed. 1981.

22. Johanson, D.; Lovejoy, C.O.; Kimbel, W.H.; White, T.D.; Ward, S.C.; Bush, M.e.; Latimer, B.M.; Coppens, Y. Morphology of the Pliocene Partial Hominoid Skeleton (AL. 288.1) fron the Hadar Formation, Ethiopia. Am.J. Physic.Anthropol. 1982; 57: 403-451.

23. Jungers, W.L. Relative Joint Size and Hominoid Locomotor Adaptations wit Implications for the Evolution of Hominid Bipedalism. J. Human. Evol. 1988; 17: 247-265.

24. Lamy, P. The Settlement of the Longitudinal Plantar Arch of Some African Plio-Pleistocene Hominids: a Morphologycal Study. J. Human. Evol. 1986; 15: 31-46.

25. Langdon, J.H. Fossils and the Origin of Bipedalism. J.Human.Evol. 1985; 14: 615-635.

26. Latimer, B.; Ohman, J.C.; Lovejoy, C.O. Talocrural Joiny in African Hominoids: Implications for Austrolopithecus Afarensis. Am.J.Phys.Anthropol. 1987; 74: $155-175$.

27. Latimer, B.; Lovejoy, C.O.; Johanson, D.; Coppens, Y. Hominid Tarsal, Metatarsal and Phalangeal Bones Recovered from the Hadar Formation: 1974-1977 Collect. Am.J.Phys.Anthropol. 1982; 57: 701-719.

28. Latimer, B.; Lovejoy, C.O. The Calceneous of Austrolopithecus Afarensis and its Implications for the Evolution of Bipedality. Am.J.Phys.Anthropol. 1989; 78: $369 / 386$.

29. Leakey, R.; Walker, A. Homo Erectur Unearthed: a Fosil Skeleton 1.6 My Old. National Geographic. 1985; 168: 5 624-629.

30. Leutenegger, W. Origin of Hominid Bipedalism. Nature 1987; 325: 305 .

31. Lovejoy, C.O. The Origin Of Man. Science. 1981; 211: 341-350.

32. Lumley, H.; Martin Bueno, M. Origen y Evolución del Hombre. Ed. Ministerio Cultura./Madrid 1984.

33. McHenry, H.M. The First Bipeds: a Comparison of the A. Afarensis and A.Africanus Postcranium and Implication for the Evolution of Bipedalism. J.Human.Evol. 1986; 15: 177191.

34. Morton, D.J. Evolution of the Man's Erect Posature. J. Morphol. Physiol. 1926; 43: 147-149.

35. Norman, D. The Illustrated Encyclopedia of Dinonosaurs. Crescent Books/NY. 1985.

36. Olson, T.R.; Siedel, M.R. The Evolutionary Basis of some Clinical Disorders of Human Foot: a Comparison Survey of the Living Primates Foot-Ankle. 1983; 3: 322-341.

37. Pickford, M. A New Look ay Kenyapithecus based on Recent Collections from Westwrn Kenya. J.Human.Evol. 1985; 14: 113-143.

38. Pickford, M. Pre-Hominids. Hominidae: Proceeding of the 2nd Int. Congress of Human Paleontology. 22/33 Ed. Jaca Book/Milan 1989. 
39. Prost, J.H. Origin of Bipedalism. Am.J.Phys.Anthropol. 1980; 52: 175-189.

40. Rak, Y. Lucy's Pelvic Anatomy: its role in bipedal Gait. J.Human.Evol. 1991; 20: 283-290.

41. Rodman, P.S.; McHenry, H.M. Bioenergetics and the Origin of Hominid Bipedalism. Am.J.Phys.Anthropol. k1980; 52: 103-106.

42. Rollison, J.; Marin, R.D. Comparative Astects of Primate Locomotion, with Special Reference to Arboreal Cercopithecines. Symp. Zool. Soc. London. 1981; 48: 377427.

43. Rose, M. Food Adquisition and The Evolution of Positional Behaviour. In Food acquisition and processing in Primates. (Plenum, NY) 1984.

44. Senut, B. Reflexions sur la Brachiation et l'Origine desd Hominides a la lumiere des Hominoides Miocenes et des Hominoides Plio-Plestocenes. Geobios. 1982; 6: 335-344.

45. Senut, B. La Locomotion des Pre-Hominides. In Hominnidae: proceedings of the 2nd Inter.Congress of Human Paleontology. 53/60 Ed. Jaca Book/Milan 1989.

46. Sinclair, A.R.E.; Leakey, M.D.; Norton-Griffiths, M. Migration and Hominid Bipedalism, Nature. 1986; 324: 307308 .

48. Smith, F.H.; Falsetti, A.B.; Donnelly, S.M. Modern Human Origins. Yearbook of Phys. Anthropol, 1989; 32: 3568.

49. Stern, J.T.; Susman, R.L. The Locomotor Anatomy of Austrolopithecus Afarensis, Am. J. Phys. Anthropol. 1983; 60: 279-317.

50. Szalay, F.S.; Langdon, J.H. The Foot of Oreopithecus: an Evolutionary Assessment. J.Human.Evol. 1986; 15: 585-
621.

51. Tobias, Ph.V. The Status of Homo Habilis in 1987 and some outstanding problems. In Hominidae: Proceeding of the 2nd Inter.Congress of Human Paleontology. Ed.Jaca Book/milan 1989.

52. Tuttle, R.S. Knucle-Walking and the Problem of Human Origins Science. 1969; 166: 953-961.

53. Tuttlke, R.S. Evolution of Hominid Bipedalism and Prehensile Capabilities. Phil.Trans.R.Soc.London. 1981; B292: 89-94.

54. Verhaegen, M. Origin of Hominid Bipedalism. Nature. 1987; 325: 305 (II).

55. Viladot, A. La Evolución del Pie

56. Washburn, S.L. Beahavior and the Origin of Man. Proc.R.Anthropol.Soc. 1967; 21-27.

57. Waver, K.F. The Search of Our Ancestors: Stones, Bones and Early Man. Nat.Geographic. 1985; 168: 5 560-623.

58. Wheeler, P.E. The Evolution of Bipedality and Loss of Functional Body Hair in Hominids. J.Human.Evol. 1984; 13: 91-98.

59. White, T.D.; Suwa, G. Homind Footprints at Laetoli: Facts and Interpretations. Am.J.Phys.Anthropol. 1987; 72: 485-514.

60. Wolpoff, M.H. Lucy's Lower Limb: long enough for Lucy to be fully Bipedal? Nature $1983 ; 304: 59-61$.

61. Wrangham, R.W. Bipedal Locomotion as a Feeding Adaptation in Gelada Baboons, and its Implications for Hominid Evolution. J.Human.Evol. 1980; 9: 329-331.

62. Zilhman, A.; Brunker, L. Hominid Bipedalism: then and now. Yearbook Phys.Anthropol. 1979; 22: 132-162. 